\title{
Tissue-specific stem cells: friend or foe?
}

\author{
Joerg Huelsken $^{1}$ \\ ${ }^{I}$ Ecole Polytechnique Fédérale de Lausanne (EPFL), ISREC (Swiss Institute for Experimental Cancer Research), National Center of \\ Competence in Research (NCCR) "Molecular Oncology", Chemin des Boveresses 155, 1066 Epalinges, Switzerland \\ Cell Research (2009) 19:279-281. doi: 10.1038/cr.2009.24; published online 2 March 2009
}

In the face of a hostile environment, the integrity of many tissues in the adult organism is maintained by a constant replacement of cells. This involves a hierarchical organization of the tissue with rare multi-potent stem cells giving rise to proliferating cells of limited proliferative capacity which in turn produce differentiating cells. While these stem cells are essential to maintain tissue homeostasis, several recent reports describe them as a major target of oncogenic mutation. Importantly, stem cells can be transformed to form benign or cancerous lesions by the action of only one oncogene or the loss of one tumor suppressor gene. This demonstrates that the regenerative capacity of stem cells comes at a price: an increased hazard of cancerogenesis. Accordingly, tissue-specific stem cells appear to directly give rise to cancer stem cells which drive tumor formation and are currently under intense investigation as interesting new targets for cancer therapy.

In the intestine, the epithelial layer covering the surface of the intestinal lumen regenerates rapidly. As in other tissues, this regeneration is controlled by a hierarchy of cells with increasing commitment to differentiation and simultaneously decreasing proliferative capacity. Tissue-specific stem cells constitute the basis of this system and

Correspondence: Joerg Huelsken E-mail: joerg.huelsken@epfl.ch possess the ability to self-renew for the life span of the organ. They give rise to rapidly cycling transit-amplifying cells which in turn generate non-cycling, terminally differentiated cells with discrete functions. Cell renewal involves continuous cell migration starting in crypts at the base of the epithelium and continuing upwards toward the tip of the villi or the luminal surface. These crypts harbor the pool of rapidly proliferating cells which can divide only a few times before producing the four differentiated cell types of the epithelium: enterocytes, paneth cells, goblet cells and enteroendocrine cells. The crypts also contain a few long-lived, multipotent intestinal stem cells. Initially, these stem cells have been identified based on their slow cycling nature in long-term label-retaining assays which rely on labeling the DNA of all dividing cells in juvenile animals followed by a chase period in which all rapidly dividing cells lose their label [1]. However, this was based on the invalidated assumption that indeed all types of stem cells divide infrequently and was not supported by lineage tracing experiments of these cells.

In the last year, we have seen enormous progress in this research area and several markers of intestinal stem cells have been identified in the mouse. For three of these markers, Bmi1, Lgr5 and Prom1 [2-4], we meanwhile have compelling evidence which confirms that the marker-expressing cells behave as true stem cells according to three criteria: long-term lineage tracing demonstrating their self-renewal capacity, labeling of the progeny with differentiation markers to demonstrate multi-potency and, at least in one case, cell-ablation experiments to prove that these stem cells are essential for crypt maintenance. All these experiments make use of knock-in alleles into the marker locus to express an inducible form of the crerecombinase under the control of the respective marker gene promoter. In combination with cre-inducible reporter alleles expressing lacZ or fluorescent proteins this system allows genetically labeling of the progeny of the stem cell. Alternatively, the combination with a cre-inducible expression of diptheria toxin has been used for cell ablation experiments in order to assess the importance of the particular stem cell population for tissue maintenance. Two of these markers, Lgr5 and Prom1, have been analyzed for the human system as well [5].

The Polycomb group protein Bmil is involved in regulating chromatin silencing and functions in the selfrenewal of neuronal, hematopoietic and leukemic stem cells. Work by the Capecchi lab has identified Bmil as a specific marker of intestinal stem cells of the anterior part of the small intestine (duodenum and jejunum) and it has been postulated that some of its close relatives might be expressed in stem cells of other parts of the intestine [2]. In comparison, the G-protein coupled receptor Lgr5, which was identified 
by the Clevers lab, is a marker which specifically labels stem cells of the whole gastro-intestinal tract [6]. Prom1 expression overlaps with Lgr5 expression in the small intestine, however, this is not the case for stem cells of the colon ${ }^{4}$. Interestingly, the $\mathrm{Bmil}^{+}$cells are localized to a region just above Paneth cells around position +4 relative to the crypt base and this localization overlaps with earlier results of long-term labelretaining cells [1]. In contrast, Lgr5 and Prom1 mark stem cells which reside at the base of the crypt intermingled with Paneth cells, and these stem cells have been termed crypt base columnar cells (CBC) [6]. Another distinguishing feature between these two populations of stem cells is their different cell cycle kinetics. Whereas Bmi1 ${ }^{+}$cells appear to divide slowly, which is in line with the properties of label-retaining cells, Lgr $^{+}$ CBCs proliferate every $24 \mathrm{~h}$. Currently, it is not clear how these two populations are interconnected and one can only speculate that the $\mathrm{Bmil}^{+}$population might be the progenitor of the $\operatorname{Lr} 5^{+}$ population, similar to the hematopoietic system where the slowest cycling cells display the most extended reconstitution capacity.

While normal tissue-specific stem cells provide the basis of tissue homeostasis, a similar hierarchical organization has been identified in tumors which frequently contain a small subpopulation of cells termed cancer stem cells. Like their normal counterparts, cancer stem cells possess the ability for longterm self-renewal while the remainder of tumor cells undergoes a process of aberrant terminal differentiation. This enables cancer stem cells to drive tumor maintenance and allows efficient tumor initiation in transplantation experiments. Such cancer stem cells were initially identified in leukemia, where the causative chromosomal translocations are present in hematopoietic stem cells while the transformed phenotype manifests in more differentiated progeny. Cancer stem cells were subsequently de- scribed in many solid tumors originating from brain, lung, breast, prostate, liver, pancreas or colon. However, there are currently only few studies which have addressed the question whether such epithelial cancer stem cells are directly derived from normal tissue-specific stem cells [7] or if they evolve from more differentiated cells which regain stem cell properties. All three studies on intestinal stem cells lend further support to the first scenario by demonstrating that specific over-activation of Wnt signaling, a well established pathway for the initiation of intestinal tumors, in intestinal stem cells can cause very rapid and efficient tumorigenesis [2-4]. Since there is also evidence for more differentiated progenitors as targets of mutation in other tissues [8], this illustrates that a certain match of mutation and target cells is required for the initiation of tumorigenesis. In such a case, a single mutation can be sufficient to generate a cancer stem cell and to allow for benign adenoma formation and additional mutations are only necessary for progression to more aggressive and invasive cancers. Interestingly, both $\mathrm{Lgr}^{+}$and Prom $1^{+}$continue to be expressed only in a small sub-population of tumor cells which suggests that these proteins might be cancer stem cells markers. While none of these studies have performed experiments to characterize the murine intestinal cancer stem cells, indirect evidence supporting this view comes from experiments with human colon cancer specimen where Prom1/CD133 has been used successfully to isolate cancer stem cells [9].

Importantly, the Clevers lab proceeded to show that the mutation which causes tumor formation is only sufficient when present in intestinal stem cells, however, not when present in other intestinal epithelial cells. Even rapidly dividing transit-amplifying cells of the crypt can not form tumors upon activation of Wnt signaling. Instead after a few initial divisions, mutant cells form small, latent clones or micro-adenomas, which persist for a long time but appear to require additional mutations for progression. This is supported indirectly by experiments from the Gilbertson lab which report that Prom1-mediated activation of Wnt signaling induces tumor formation only in the small intestine but not in the colon, presumably since Prom 1 is not expressed in stem cells of the colon. What do these experiments tell us about the role of the Wnt pathway in inducing intestinal tumors? In line with previous experiments in skin tumors [7], these data support the concept that Wnt signaling prevents differentiation of intestinal stem or transit-amplifying cells and sustains their undifferentiated state, instead of stimulating their proliferation. A block in the pathway induces rapid terminal differentiation of stem cells [10], and an over-activation produces cells with an aberrant transit-amplifying phenotype which are characterized by a largely expanded self-renewal capacity. This suggests that a better understanding about the molecular signals which are responsible for controling long-term self-renewal vs. limited cell cycling could provide new insights to aid therapeutic design for the treatment of colon cancer patients.

\section{References}

1 Potten CS, Owen G, Booth D. Intestinal stem cells protect their genome by selective segregation of template DNA strands. J Cell Sci 2002; 115:23812388.

2 Sangiorgi E, Capecchi MR. Bmil is expressed in vivo in intestinal stem cells. Nat Genet 2008; 40:915-920.

3 Barker N, Ridgway RA, van Es JH, et al. Crypt stem cells as the cells-of-origin of intestinal cancer. Nature 2009; 457:608611.

4 Zhu L, Gibson P, Currle DS, et al. Prominin 1 marks intestinal stem cells that are susceptible to neoplastic transformation. Nature 2009; 457:603-607.

5 Becker L, Huang Q, Mashimo H. Immunostaining of Lgr5, an intestinal stem cell marker, in normal and premalignant human gastrointestinal tissue. Scientific- 
WorldJournal 2008; 8:1168-1176.

6 Barker N, van Es JH, Kuipers J, et al. Identification of stem cells in small intestine and colon by marker gene Lgr5. Nature 2007; 449:1003-1007.

7 Malanchi I, Peinado H, Kassen D, et al. Cutaneous cancer stem cell maintenance is dependent on $\beta$-catenin signaling.
Nature 2008; 452:650-653.

8 Castor A, Nilsson L, Astrand-Grundström I, et al. Distinct patterns of hematopoietic stem cell involvement in acute lymphoblastic leukemia. Nat Med 2005; 11:630-637.

9 O'Brien CA, Pollett A, Gallinger S, Dick JE. A human colon cancer cell capable of initiating tumour growth in immunodeficient mice. Nature 2007; 445:106-110.

10 Fevr T, Robine S, Louvard D, Huelsken J. Wnt/ $\beta$-Catenin Is Essential for Intestinal Homeostasis and Maintenance of Intestinal Stem Cells. Mol Cell Biol 2007; 27:7551-7559. 